\title{
A SEARCH FOR VARIABLES IN THE CENTRAL REGIONS OF SOUTHERN GLOBULAR CLUSTERS
}

\author{
J. W. MENZIES
}

South African Astronomical Observatory, PO Box 9, Observatory 7935, South Africa

\begin{abstract}
Preliminary results are presented for 3 southern globular clusters that have been searched via CCD photometry for new short-period variables that might be associated with blue stragglers. The clusters considered here are NGC6121, NGC3201 and NGC6809.
\end{abstract}

\section{NGC6121}

From a number of observational series, one series in each of $\mathrm{V}$ (58 frames) and I (65 frames) has been measured. A 3' $\mathrm{x} 2$ ' region including the cluster centre has been observed. Three new contact binaries have been found, with probable periods in the region of 0.3 to 0.4 day. One of them is clearly a blue straggler, the second lies just beyond the turnoff while the third is very red, lying below the subgiant branch.

\section{NGC3201}

A search was made on 2 series of images covering areas of 3 ' $\mathrm{x} 2$ ' in the northern ( $23 \mathrm{~V}$ frames) and southerni (19 V frames) halves of the cluster. The cluster centre was on the edge of the frames for each series. Apart from the already known RR Lyrae stars, no variables were found with amplitudes greater than $0.1 \mathrm{mag}$.

\section{NGC6809}

A 3'x 2' field centred on the cluster centre has been surveyed for variables. Apart from the known RR Lyrae stars, one new eclipsing variable has been found with a primary eclipse depth of $1.8 \mathrm{mag}$. Altogether, $311 \mathrm{~V}$ 
frames obtained in 5 observing weeks were measured. A possible secondary minimum of depth about $0.1 \mathrm{mag}$ was seen Rin one series. No other variables with amplitudes greater than $0.1 \mathrm{mag}$. The eclipsing variable has a period of 1.209day, and lies amongst the blue stragglers in the colour-magnitude diagram. 